\title{
3D fluorescence emission difference microscopy based on spatial light modulator
}

\author{
Guangyuan Zhao, Zihao Rong, Cuifang Kuang*, Cheng Zheng and Xu Liu ${ }^{\dagger}$ \\ State Key Laboratory of Modern Optical Instrumentation \\ College of Optical Science and Engineering \\ Zhejiang University, Hangzhou 310027, P. R. China \\ *Cfkuang@zju.edu.cn \\ ${ }^{\dagger}$ Liuxu@zju.edu.cn
}

Received 28 February 2016

Accepted 6 April 2016

Published 17 May 2016

\begin{abstract}
We report three-dimensional fluorescence emission difference (3D-FED) microscopy using a spatial light modulator (SLM). Zero phase, $0-2 \pi$ vortex phase and binary 0-pi phase are loaded on the SLM to generate the corresponding solid, doughnut and $z$-axis hollow excitation spot, respectively. Our technique achieves super-resolved image by subtracting three differently acquired images with proper subtractive factors. Detailed theoretical analysis and simulation tests are proceeded to testify the performance of 3D-FED. Also, the improvement of lateral and axial resolution is demonstrated by imaging $100 \mathrm{~nm}$ fluorescent beads. The experiment yields lateral resolution of $140 \mathrm{~nm}$ and axial resolution of approximate $380 \mathrm{~nm}$.
\end{abstract}

Keywords: Super-resolution; fluorescence imaging; 3D-FED microscopy; spatial light modulator.

\section{Introduction}

Far-field fluorescence microscopy has been applied in biological and medical science to observe the microstructures and their movements ${ }^{1}$ owing to its simplicity, versatility, and noninvasiveness. However, the diffraction barrier ${ }^{2}$ confines the resolving ability of conventional far-field fluorescence microscopy to about half the illumination light wavelength, restricting observation of microstructures featuring length scales of less than $100 \mathrm{~nm}$, such as vimentin fibers, ${ }^{3}$ microtubules, and vesicles.
Confocal scanning laser microscopy $(\mathrm{CSLM})^{4}$ can enhance the spatial resolution by a factor of $\sqrt{2}$ and improve micrograph contrast by spatial filtering with a pinhole, which also endows CSLM with optical sectioning ability. ${ }^{5}$ Nonetheless, CSLM resolution still cannot break the diffraction barrier.

Fluorescence emission difference $(\mathrm{FED})^{6,7} \mathrm{mi}-$ croscopy was recently reported as a novel superresolution technique. It is based on intensity subtraction $^{8}$ between two images acquired under solid and hollow illumination patterns. It joins a

*Corresponding author.

This is an Open Access article published by World Scientific Publishing Company. It is distributed under the terms of the Creative Commons Attribution 4.0 (CC-BY) License. Further distribution of this work is permitted, provided the original work is properly cited. 
long list of validated super-resolution fluorescence microscopy methods, e.g., stochastic optical reconstruction microscopy (STORM), ${ }^{9}$ photoactivated localization microscopy (PALM) ${ }^{10}$ stimulated emission depletion microscopy (STED), ${ }^{11}$ structured illumination microscopy (SIM) ${ }^{12,13}$ digital image processing, ${ }^{14,15}$ and total-internal reflection microscopy based on a nanostructured substrate. ${ }^{16}$ STED uses a solid focal spot to stimulate fluorescence emission and a hollow spot to deplete the margin area of fluorescence spot, thus reducing the size of fluorescence spot physically, while FED fulfills the similar process mathematically. Similar methods include switching laser mode microscopy $(\mathrm{SLAM})^{17}$ and ground state depletion microscopy (GSD). ${ }^{18}$ Without the depletion process, FED has some advantages over STED: low power, simple optical setup and wide variety of available dyes, which undoubtedly add to its availability in practical scientific research. ${ }^{19}$

A 3D image can be produced by scanning through the whole space in the conventional confocal methods, however former researches for FED and SLAM are mostly concentrated on the enhancement of lateral resolution ${ }^{6,17,20-23}$ but the axial resolution is not enhanced.

In this work, we present a simple approach to achieve 3D super-resolution imaging using FED, and we call it the $3 \mathrm{D}-\mathrm{FED}$. Zero phase, $0-2 \pi$ vortex phase and binary 0-pi phase are loaded on the spatial light modulator (SLM) to generate the corresponding solid, doughnut, and $z$-axis hollow excitation spot, respectively. Our technique achieves super-resolved image by subtracting three differently acquired images with the proper factors according to the simulation and experiment situation. ${ }^{19,24-27}$ Images of fluorescent beads on a coverslip prove that this technique can be implemented successfully in confocal microscopy and improves not only the lateral but also the axial resolution.

\section{Theory}

In 3D-FED, three different confocal scanning images are required to obtain the final 3D-FED image: the confocal image is acquired under the solid excitation pattern, the lateral negative confocal image is acquired under the doughnut excitation pattern, ${ }^{7}$ and the axial-negative confocal image is acquired under the $z$-axis hollow excitation pattern. The excited fluorescence is filtered by a pinhole and detected by a photomultiplier tube (PMT) to form images. The final 3D-FED super-resolution image is constructed by mathematical intensity subtraction of the three stacks of images.

$$
I_{3 \mathrm{dFED}}=I_{\text {solid }}-r \times I_{\text {dnegative }}-q \times I_{\text {znegative }} .
$$

In this equation, $I_{3 \mathrm{dFED}}, I_{\text {solid }}, I_{\text {dnegaitve }}, I_{\text {znegative }}$ are the normalized intensity distributions of the 3DFED, 3D-Confocal, lateral-negative confocal, and the axial-negative confocal images, respectively, $r$ and $q$ denote the subtraction factors. Negative intensity values which are inevitable after subtraction are excluded from the final image for the sake of a better imaging quality.

Surely, as long as the system of 3D-FED is similar to that of confocal microscopy, the background noise of each image is also the same with confocal images. However, the background noise is reduced by subtraction because the three confocal images are taken in the same environment and have the same background noise, hence 3D-FED improve the signal-to-noise ratio (SNR) compared to confocal method.

The resolving ability of 3D-FED is determined by the point spread functions (PSFs) of the three illumination modes which can be described with Debye integral, ${ }^{20,28,29}$

$$
\begin{aligned}
\mathbf{E}\left(r_{2}, \varphi_{2}, z_{2}\right) & \\
= & i C \iint_{\Omega} \sin \theta \cdot A_{1}(\theta, \varphi) \cdot A_{2}(\theta, \varphi) \\
& \cdot\left[\begin{array}{c}
p_{x} \\
p_{y} \\
p_{z}
\end{array}\right] \cdot \exp [i f(\theta, \varphi)] \cdot \exp \left[i k n \left(z_{2} \cos \theta\right.\right. \\
& \left.\left.+r_{2} \sin \theta \cos \left(\varphi-\varphi_{2}\right)\right)\right] d \theta d \varphi .
\end{aligned}
$$

In the integral, $\mathbf{E}\left(r_{2}, \varphi_{2}, z_{2}\right)$ refers to the electric field vector at the point $\left(r_{2}, \varphi_{2}, z_{2}\right)$ in cylindrical coordinates with its origin at the focal point of the objective lens (OL). $(\theta, \varphi)$ represents the position of the individual points on the wave front of the incident beam, while $\theta$ is the angle between the ray direction and the optical axis and $\varphi$ is the azimuthal angle. $\Omega$ stands for the effective incident aperture of the beam, $A_{1}(\theta, \varphi)$ denotes the amplitude function of the input light, and $A_{2}(\theta, \varphi)$ is the aberration function determined by the structure of the OL. $\left[p_{x}, p_{y}, p_{z}\right]^{T}$ stands for the polarization state of the incident beam and $f(\theta, \varphi)$ represents the phase modulation function applied to the input light. We 
set $z$-axis as the optical axis of the OL throughout this paper. According to the variables in this equation, we obtained three kinds of focal spot by tuning the phase distribution. The first kind is ordinary confocal spot without beam modulation. The second kind is generated by $0-2 \pi$ vortex phase plate modulation called laterally hollow spot. The third kind is generated by binary $0-\pi$ phase plate modulation called axial hollow spot. Phase delay functions of the three patterns of illumination are as follows:

$$
\begin{gathered}
\Delta \alpha_{1}(\theta, \varphi)=0 \\
\Delta \alpha_{2}(\theta, \varphi)=\phi \\
\Delta \alpha_{3}(\theta, \varphi)=\left\{\begin{array}{l}
\pi, \theta<\frac{\sqrt{2}}{2} \theta_{\max } \\
0, \theta \geq \frac{\sqrt{2}}{2} \theta_{\max }
\end{array}\right.
\end{gathered}
$$

The PSF of 3D-FED is given by:

$$
\begin{aligned}
\mathrm{PSF}_{3 \mathrm{dFED}} & \\
= & \mathrm{PSF}_{s} \times\left(\mathrm{PSF}_{f} \otimes p\right)-r \times \mathrm{PSF}_{d} \\
& \times\left(\mathrm{PSF}_{f} \otimes p\right)-q \times \mathrm{PSF}_{z} \times\left(\mathrm{PSF}_{f} \otimes p\right) .
\end{aligned}
$$

Here, the $\mathrm{PSF}_{3 \mathrm{dFED}}, \mathrm{PSF}_{s}, \mathrm{PSF}_{d}, \mathrm{PSF}_{z}$, and $\mathrm{PSF}_{f}$ are the PSFs for 3D-FED imaging, solid spot, lateral hollow spot, axial hollow spot, and fluorescence collection spot. $p$ denotes the $2 \mathrm{D}$ transmission function of the pinhole. PSF for a fluorescence emission spot is identical to the intensity distribution of illumination spot when neglecting fluorescence saturation.

Equations (1)-(5) mentioned above are the theoretical basis of 3D-FED. We can obtain the PSF of three different patterns of emission spots by using (Eqs. 2-5). The calculated intensity distributions with different phase patterns and the corresponding $3 \mathrm{D}-\mathrm{FED}$ results are shown in Fig. 1 . The region of $3 \mathrm{D}$ space is $2 \lambda \times 2 \lambda \times 2 \lambda$, Figs. 1 (a) -1 c denote the $0,0 \sim 2$ pi vortex and binary 0 -pi phase patterns loaded on the SLM. $Z$-axis is set as the optical axis. When we switch the phase pattern, we can obtain the solid spot, lateral-hollow spot, and axial-hollow spot, alternatively. Figures 1(d)-1(f) illustrates the $x y$ plane PSFs of the 3D solid spot, 3D $x y$-hollow spot, 3D $z$-hollow spot. Also, Figs. 1(g)-1(i) show the $x-z$ plane PSFs of the $3 \mathrm{D}$ spots. Then we use Eq. (1) to process the subtraction, the subtractive factor $r$ is $0.7, q$ is 0.5 , the negative value is set to be zero. Figure $1(\mathrm{j})$ is the lateral super-resolution image of 3D-FED results and Fig. 1(k) is the axial super-resolution image of it. Obviously, by comparing the lateral profiles of confocal image in Fig. 1 (d) and the 3D-FED image in Fig. 1(j), we can easily discover that the effective PSF has been reduced. The same conclusion can be drawn when we compare the axial profiles of 3D-FED and 3D confocal images, which means, the 3D-FED method can be used to enhance the confocal resolution both axially and laterally.

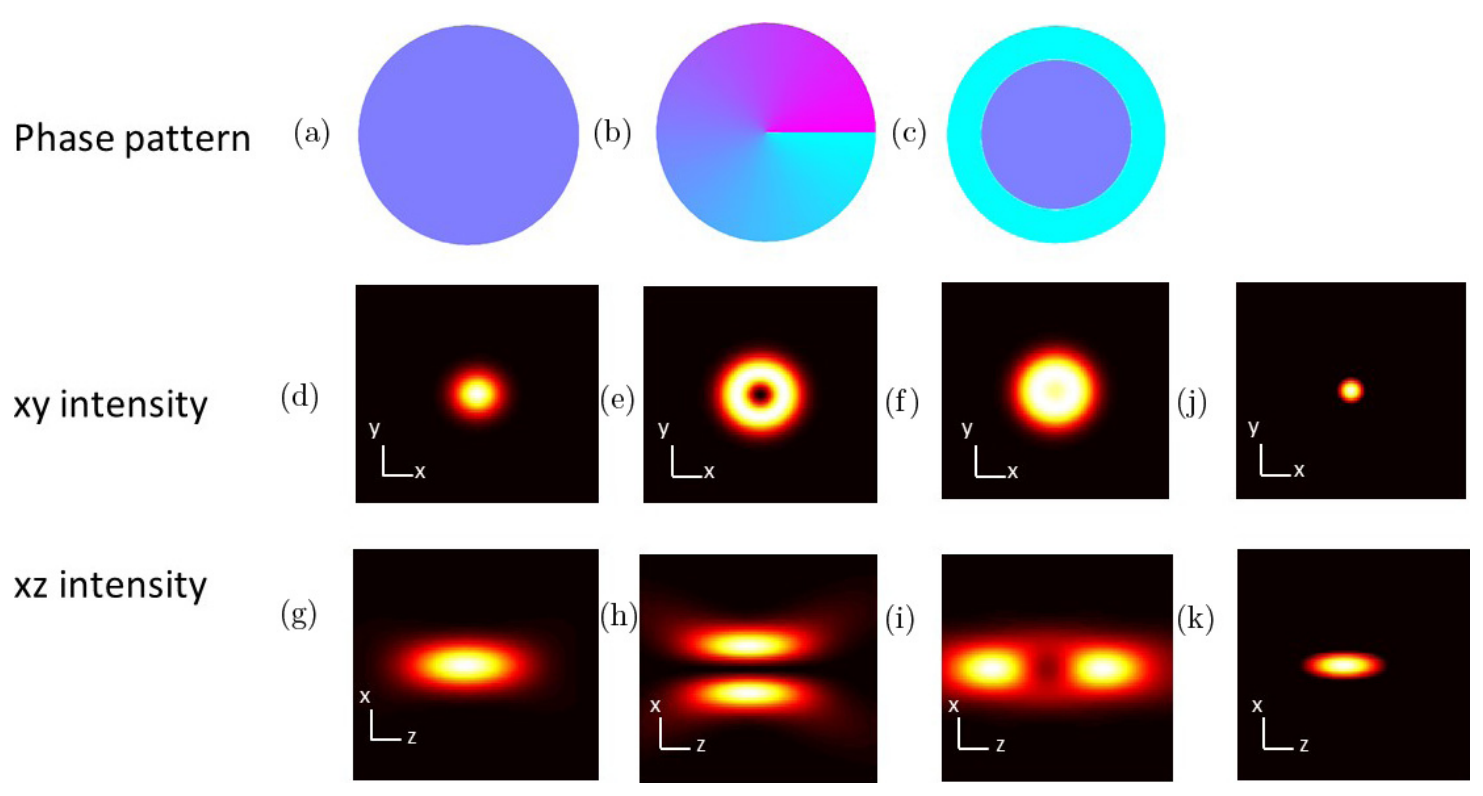

Fig. 1. Phase patterns and the corresponding normalized intensity distribution. 


\section{Multipoint Simulation}

To further demonstrate the resolving ability enhancement of the 3D-FED, we designed a $3 \times 3 \times 3$ point lattice sample in a $4 \lambda \times 4 \lambda \times 4 \lambda$ cube. The lateral distance between the adjacent points is $0.4 \lambda$ and the axial adjacent points are separated by a distance of $0.8 \lambda$. The PSFs are calculated by Debye integrals using MATLAB and the system parameters are shown in Table 1. Then we obtain 3DFED images using Eq. (1). The axial and lateral images of conventional 3D-confocal microscope are shown in Figs. 2(a) and 2(c), from which we can see that the points in $x-y$ slice cannot be clearly resolved and the points along the axial axis can be hardly discerned. Figures 2(b) and 2(d) show the lateral and axial imaging results by our 3D-FED method. It is noted that all the points in the lateral image can be completely resolved in Fig. 2(b). Also, the resolution of axial point is improved dramatically without obvious distortion. Figures $2(\mathrm{e})$ and 2 (f) illustrate the 3D display of simulation results of confocal and 3D-FED method. In Fig. 2(e), the array point can hardly be discerned and appears like a cuboid, but in Fig. 2(f) the points appear separated from each other and hence be distinguished.

In order to further distinctly compare the resolution enhancement, we extracted the linear profiles of intensity along lateral position in Fig. 3(a) and axial position in Fig. 3(b) from the lateral and axial images. In Fig. 3(a), the red solid curve represented the confocal lateral linear intensity which shows that the points cannot be distinguished clearly while the linear profile of 3D-FED can distinguish them. According to the concept of Rayleigh criterion, two peaks can be discerned when the minimum magnitude between them is no more than $73.5 \%$ of the peak magnitude. Obviously, black line for 3D-FED

Table 1. System parameters for simulation.

\begin{tabular}{lc}
\hline Parameter & Value \\
\hline Side length of image scope $(\lambda)$ & $4 \times 4 \times 4$ \\
Pixels of image scope & $101 \times 101 \times 101$ \\
Size of sample (point) & $3 \times 3 \times 3$ \\
Excitation wavelength (nm) & 635 \\
Fluorescence central wavelength (nm) & 650 \\
NA & 1.4 \\
Polarization & Right hand circular \\
Refractive index & 1.518 \\
Pinhole (airy disk diameters) & 0.6 \\
Subtracted factor & $r=0.8, q=0.5$ \\
\hline
\end{tabular}

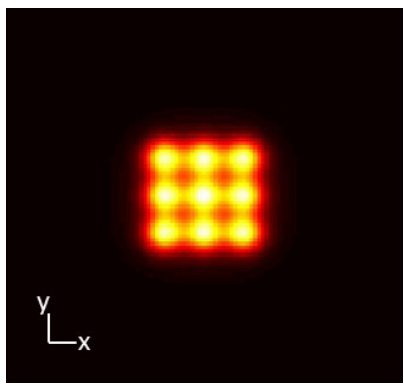

(a)

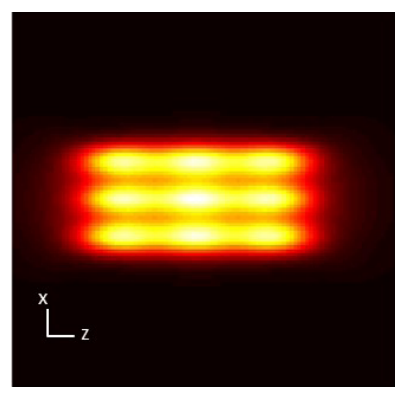

(c)

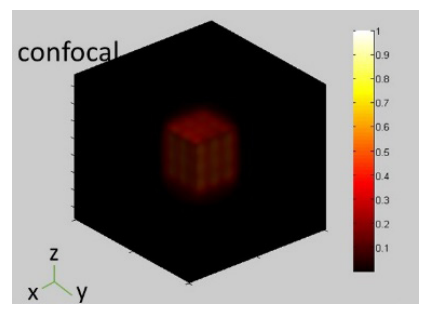

(e)

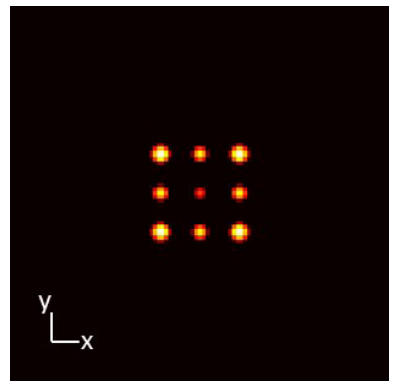

(b)

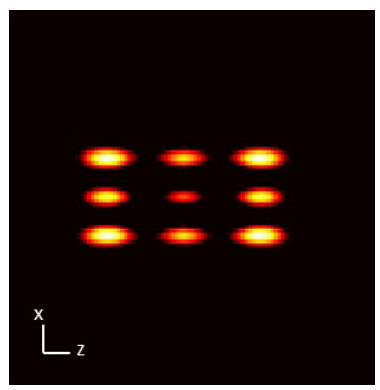

(d)

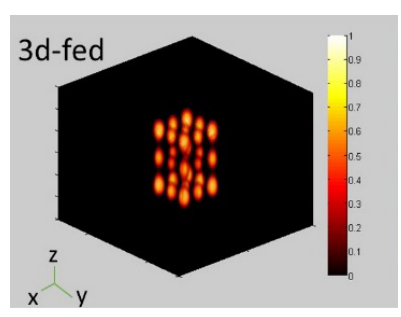

(f)
Fig. 2. Simulation results of a $3 \times 3 \times 3$ point array sample. Side length of the scope is $4 \lambda$. (a) and (c): Lateral and axial images of confocal microscope. (b) and (d): Lateral and axial images of 3D-FED microscope. (e) and (f): 3D display of confocal and 3D-FED microscope.

imaging satisfies this condition while red line for confocal imaging does not. Meanwhile, we extract the 2D-FED linear profile as shown in Fig. 3(a) from the image $I_{2 \mathrm{dFED}}$ which is obtained from the equation (the so-called conventional FED method):

$$
I_{2 \mathrm{dFED}}=I_{\text {solid }}-r \times I_{\text {dnegative }},
$$

where $I_{2 \mathrm{dFED}}, I_{\text {solid }}$, and $I_{\text {dnegative }}$ are normalized intensity distributions of subtracted results, 3Dconfocal image and 3D-doughnut confocal image generated by the vortex phase pattern. By comparing the three curve lines, we can find that the conventional FED method can also improve the axial resolution to a certain extent and meet 


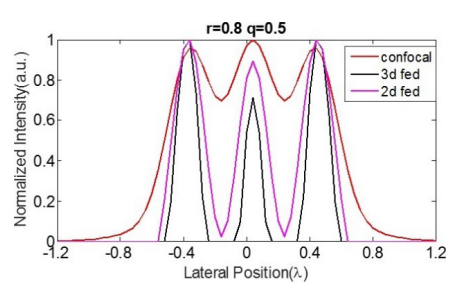

(a)

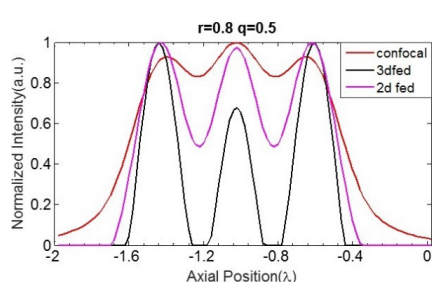

(b)

Fig. 3. Linear profile of normalized intensities. (a) Linear profile of simulation results along lateral direction. (b) Linear profile of simulation results along axial profile.

the demand of Rayleigh criterion though not as well as the 3D-FED.

As well, the pink curve line named 2D-FED in Fig. 3(b) is the axial linear profile extracted from the $I_{2 \mathrm{dFED}}$ in Eq. (7). The black curve line named 3D-FED in Fig. 3(b) denotes the axial linear profile of 3D-FED intensity along $x$-axis, while the red line denotes the axial linear profile of 3D confocal intensity along $x$-axis. We can see that the $3 \mathrm{D}$-FED can significantly improve the axial resolution.

\section{Experiment}

\subsection{Phase pattern generation with SLM}

Our new 3D-FED experimental setup is built up based on the phase only SLM to generate three kinds of time-divided phase patterns, the included angle between the light incident on the liquid crystal surface and the light reflected from the surface should be less than $8^{\circ}$. Once the sample is scanned, the phase pattern loaded on SLM changes. The new system does not need extra optical path to generate different types of phase delayed illumination light and can achieve 3D super resolution with the appropriate binary 0 -pi phase pattern.

\subsection{Experimental configuration}

Based on the concept of 3D-FED, we built a new 3D-FED experimental setup as depicted in Fig. 4. A collimating lens (CL1) is employed to collimate the laser beam emitted by a laser diode (Coherent CL 2000) at the wavelength of $633 \mathrm{~nm}$ and transmitted through a single-mode fiber. The collimated beam goes through a polarizing plate and become a linearly polarized beam of specific angle. By rotating the polarizing plate, the linearly polarizing beam is turned into $p$-polarization beam, which will then be reflected and modulated by the SLM (BNS $635 \mathrm{~nm}$ ). After the phase modulation, two $\lambda / 4$ wave plates $(\lambda / 4 \mathrm{WP})$ are used to modulate the linearly polarized beam into right hand circularly polarized beam. The circularly polarized beam then goes through a galvo mirror (Thorlabs GVS002) controlled by a DAQ card (NI USB-6259) to realize two-dimensional fast scan of the sample. The scanning lens (SL) creates an intermediate image before the tube lens (TL) parallelizing the laser light. Then a high numerical OL (Olympus Uplan SApo 100x/1.4 Oil) which is fixed on a nano positioning $z$-stage (PI E$665)$ serves to strongly focus the circularly polarized beam and collect the fluorescence emitting from the sample. The 3D scanning is then realized combining the galvo mirror and the nano positioning $z$-stage. The dichroic mirror (DM) (Chroma ZT647 rdc-uf3) which reflects the illumination while transmitting the fluorescence is then cleaned up by the bandpass filter (ET $705 / 72 \mathrm{~m})$. Next, the fluorescence is further filtered by a confocal pinhole, coupled into a multi-mode fiber (M31L02, Thorlabs). The fiber is attached to an avalanche photo diode (SPCMAQR-16-FC, PerkinElmer) which detects the intensity of the fluorescence beam. The detected fluorescence data are processed by the inspector and a set of self-written routines in MATLAB (The Mathworks, Natcik, CA).

\subsection{Results of nanoparticles}

We imaged $100 \mathrm{~nm}$ fluorescent nanoparticles (T7279, TetraSpeck ${ }^{\mathrm{TM}}$ Microspheres, $0.1 \mu \mathrm{m}$, fluorescent blue/green/orange/dark red) to verify the resolution enhancement of our 3D-FED system. The fluorescent beads are prepared without dilution because of its low concentration. In order to obtain the solid fluorescent images, doughnut fluorescent images and $z$-axis negative images, we scanned the 


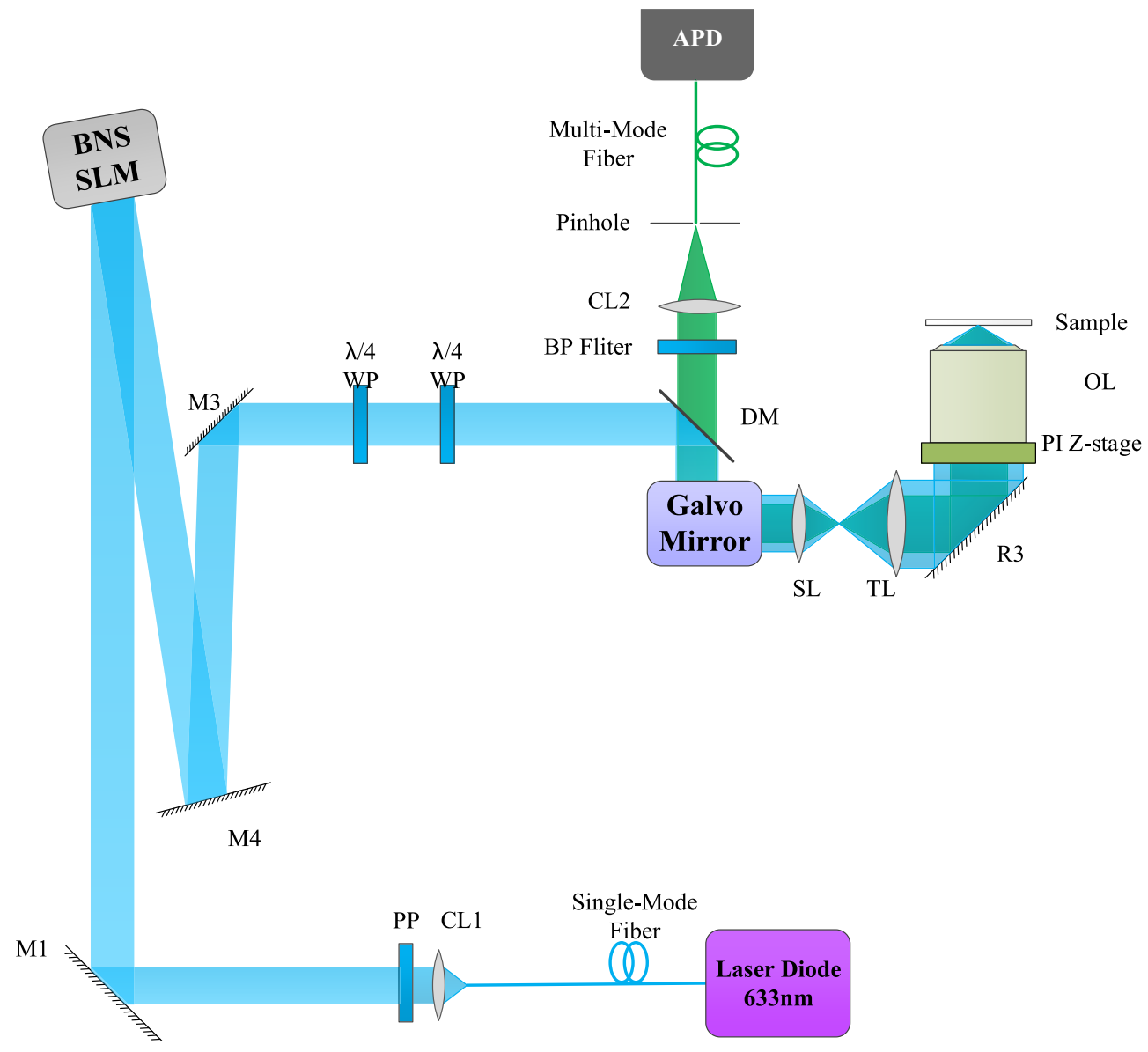

Fig. 4. Configuration of a 3D-FED microscopy system. CL1-collimating lens, $\lambda / 4 \mathrm{WP}-\lambda / 4$ wave plate, PBS-polarizing beam splitter, M-reflecting mirror, BNS SLM-BNS phase only SLM, BP filter-bandpass filter, DM, SL, TL, OL, CL2-convergent lens, APD-avalanche photon diode.

sample for three times. The 3D-FED images is obtained by processing three stacks of images according to Eq. (1) using a self-developed MATLAB software.

Negative values may appear in 3D-FED image as a result of subtraction, in order to present an acceptable image with only positive voxels, these negative values can be set to 0 . Although this operation is at the risk of discarding useful signal, which may lead to the missing of objects, it is the case of very high subtractive factor. It is clear that increasing the value of $r$ and $q$ will enhance the resolution, however the negative values will magnify by this operation. Take an example from simulation, when the values of $r$ and $q$ are set to be 0.7 and 0.5 , the corresponding normalized negative value will be -0.6 , continuous magnification of the factors will slightly enhance the resolution while tremendously magnifying the negative values. Consequently, the values of $r$ and $q$ are adjusted under the balance between negative artifacts and resolution to obtain the best quality 3D-FED images.

\subsubsection{D-FED imaging}

We first examined the lateral resolution enhancement of the new FED system using 2D analysis according to Eq. (7). The 2D experimental results of the fluorescent beads is shown in Fig. 5. Figures 5(a)-5(c) presents the confocal, FED and deconvolution-FED images. In the top right corner of the three images are magnified view of the region indicated by the white boxes in Figs. 5(a)-5(c), from which we can see that the two beads in confocal image appear stuck together and can hardly be discerned, but they are clearly separated into two nanoparticles in FED image and shows an even better resolution in the FED+ image. Figure 5(f) shows the normalized 
(a)

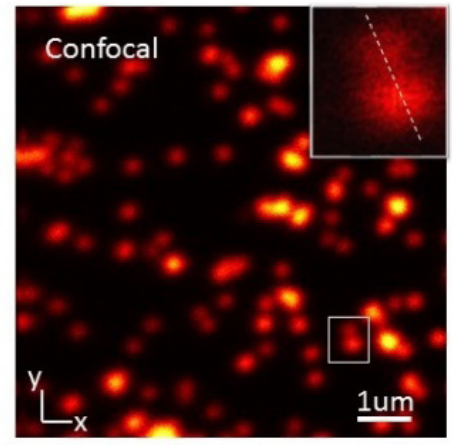

(d)

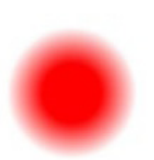

(b)

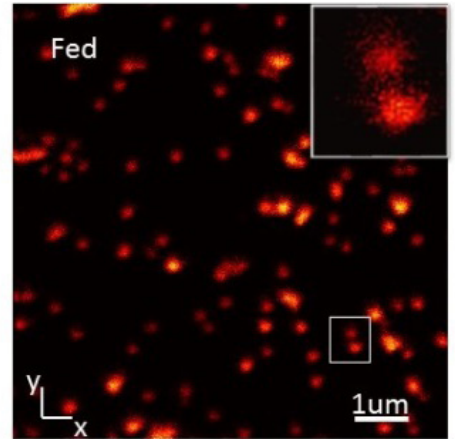

(c) Fed+

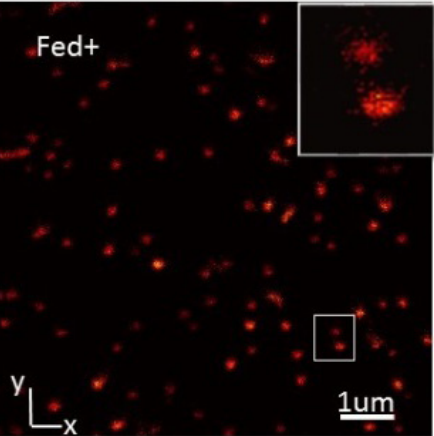

(f)

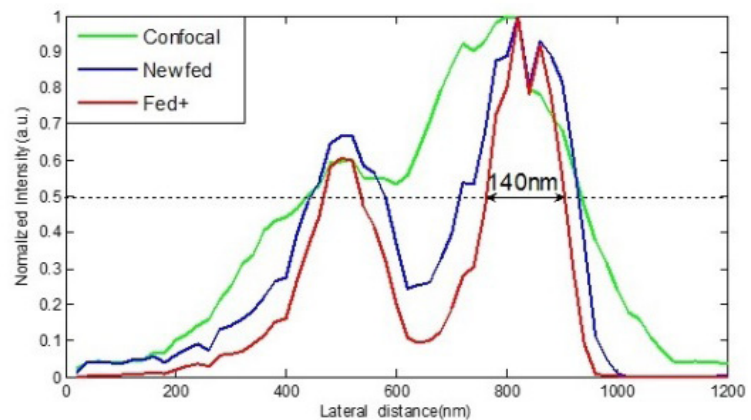

Fig. 5. 2D fluorescent nanoparticle imaging by FED and confocal methods. The $(x, y)$-scan size is $8 \times 8 \mu \mathrm{m}^{2}(512 \times 512$ pixels $)$ resulting in a total acquisition time of $\sim 5.24 \mathrm{~s}$. (a) Confocal image. (b) The new 2D-FED image with the subtractive factor $r=0.61$. (c) Corresponding deconvoluted FED image. (d) Schematic of 2D solid spots. (e) Schematic of 2D doughnut spot. (f) Lateral intensity profiles along the white dashed line in aand correspondingb, c verify the resolving ability of the 2D-FED, indicating an effective resolution of $\sim 140 \mathrm{~nm}$.

intensity profiles along the dashed line in Figs. 5(a)$5(\mathrm{c})$, the green, blue, and red lines denotes the intensity profiles in the confocal, FED and FED+ images, respectively. In confocal profile, the two particle peaks cannot be separated but in FED profile, we can obviously see the peaks and valley. The full width at half-maximum (FWHM) of FED image indicated by the double headed arrow in Fig. 5(f) can be $140 \mathrm{~nm}$, which demonstrate the lateral resolution enhancement of our FED method.

\subsubsection{D-FED imaging}

To demonstrate both axial and lateral resolution improvement, the $(x, y, z)$-stacks consisting of 40 $(x, y)$ slices of $8 \times 8 \mu \mathrm{m}^{2}(400 \times 400$ pixels $)$ are recorded, with an axial distance of $60 \mathrm{~nm}$ between adjacent slices, a lateral direction pixel size of $20 \mathrm{~nm}$ and a dwell time of $0.01 \mathrm{~ms}$ per pixel. Thus, the volume of reconstructed 3D-FED image is $8 \times 8 \times 2.4 \mu \mathrm{m}^{3}$ and corresponding scanning period is approximatly $64 \mathrm{~s}$. Lateral and axial view of the obtained confocal and 3D-FED images is shown in Fig. 6. Figure $6(\mathrm{a})$ is the lateral view of confocal image obtained by employing the 22nd slice in the stacks, in which the nanoparticles in white box overlap with each other and consequently cannot be discerned. While in Fig. 6(b), the nanoparticles are readily discernible and the beads in the image are smaller than that in Fig. 6(a). Figures 6(c) and 6(d) are the $x-z$ view of the confocal and FED images along the line in (a) and (b) with the image size of $8 \times 2.4 \mu \mathrm{m}^{2}(400 \times 120$ pixels, side length of each pixel is $20 \mathrm{~nm}$ ). By comparing the two images, we can see that the nanoparticles along the axial direction in confocal image appear stuck together and can barely be distinguished, but they are separated in the corresponding 3D-FED image. Figure 6(e) shows the intensity profiles along the direction indicated by the arrows in Figs. 6(c) and 6(d), from which we can further verify that the resolving ability is enhanced with 3D-FED. The $z$-axis profile indicated in green line in Fig. 6(d) quantifies the FWHM of axial beads can reach to about $380 \mathrm{~nm}$ comparing to the $650 \mathrm{~nm}$ of confocal images in Fig. 6(e) and break the diffraction barrier along $z$-axis to a certain degree. The results confirm that the $z$-axis resolution is improved as well. 


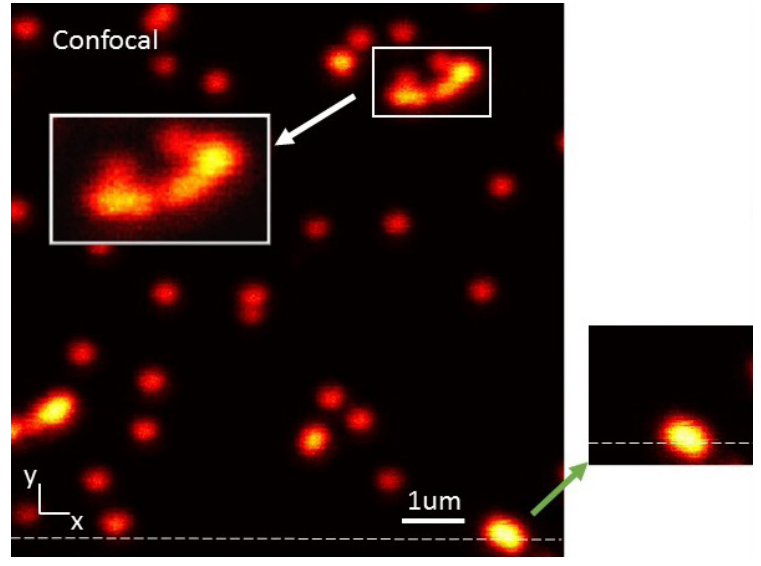

(a)

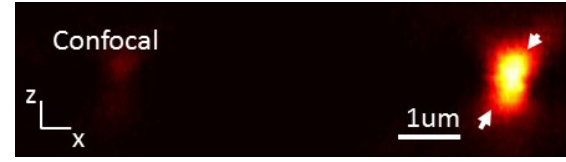

(c)

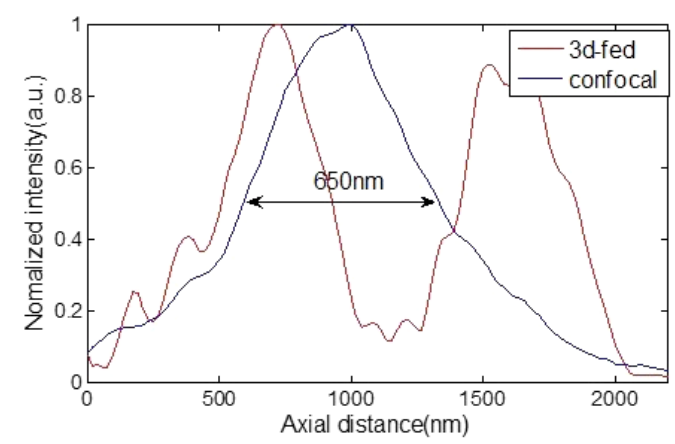

(e)

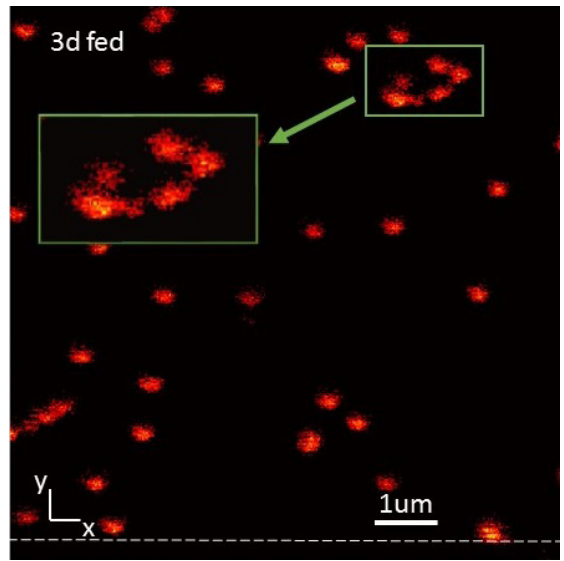

(b)

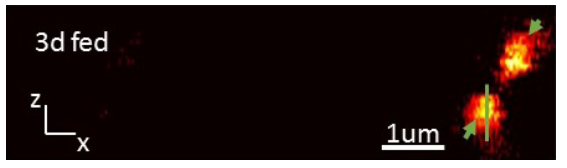

(d)

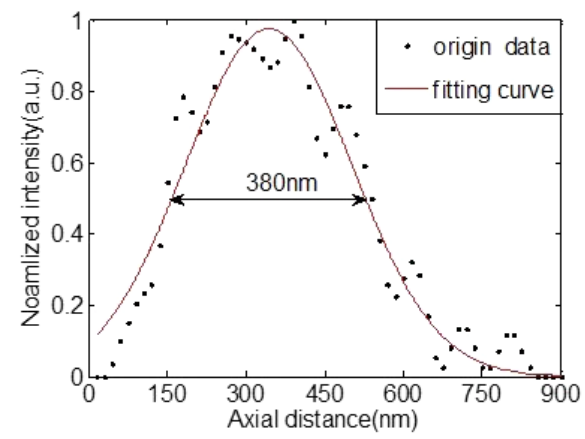

(f)

Fig. 6. Characterization of the lateral and axial resolution improvement using $0.1 \mu \mathrm{m}$ beads on a coverslip. (a), (b) $x-y$ view of confocal and 3D-FED images by employing the $22 / 40$ slices in stacks. (c) $x-z$ view of the confocal image along the dashed line in (a), in the middle of (a) and (b) is the partly magnified view of the dashed line. (d) Axial profile of 3D-FED image counterpart to (c). (e) Line profile intensity at the indicated direction in (c) and (d). (f) Intensity profiles along the $z$-axis extracted from (d) at the solid line, quantifying the axial resolution gain in 3D-FED method.

\section{Discussion and Conclusion}

In this work, we first theoretically demonstrated performance enhancement of our 3D-FED method using Debye integral calculations and MATLAB simulations. To verify the theoretical results, a 3DFED system based on our proposal is built. The subtractive factor $r$ and $q$ range from 0.4 to 1.0 empirically, depending on the practical situation. The 3D-FED system has superior resolving ability, with lateral resolution of less than $150 \mathrm{~nm}$ and axial resolution of about $380 \mathrm{~nm}$.
The confocal pinhole in our setup is composed of a multimode optical fiber (M31L02, Thorlabs) with a pinhole size $S_{\text {pinhole }}=62.5 \mu \mathrm{m}$, combing with the TL, SL, OL and convergent lens in Fig. 4. This yields a pinhole size $S_{\text {pinhole }}=0.7_{\text {Airy }}$. The pinhole size has been demonstrated to render the proper SNR and resolution in our 3D-FED system.

The sample is scanned frame by frame with the galvo mirror and the $z$-stage. We scan several $x y$ plane slices with the help of galvo mirror. When the scanning of each slice is done, the scanning plane is 
moved along the $z$-axis position by using the PI $z$-stage. The $3 \mathrm{D}$ volume is then obtained by pitching the scanned $x y$ slices together. Finally, the superresolved $3 \mathrm{D}$ volume is realized by the subtraction of three 3D volumes with different excitation modes. In the conventional FED method in which we use the phase plate to change the illumination pattern, the phase pattern does not change until every scanning is completed. Sample mismatch may be introduced and eventually cause the pixel mismatch as well as the deterioration of the image quality. By utilizing the SLM to complement the new FED experiment, we does not need to introduce additional device to change the phase every time we change the phase pattern into solid, doughnut or binary 0 -pi, only to change the phase images imposed on the software which is related to the SLM device.

In conclusion, compared with the existing 3D super-resolution methods for the simplicity and convenience, our 3D-FED microscopy has a significant resolution improvement both laterally and axially, thus we believe that such method can be developed into a useful technique.

\section{Acknowledgments}

This work was financially supported by grants from the National Basic Research Program of China (973 Program) (No. 2015CB352003), the National Natural Science Foundation of China (Nos. 61377013, 61335003,61378051 , and 61427818), NSFC of Zhejiang province LR16F050001, Innovation Joint Research Center for iCPS (2015XZZX005-01), and Open Foundation of the State Key Laboratory of Modern Optical Instrumentation.

\section{References}

1. T. A. Klar, S. W. Hell, "Subdiffraction resolution in far-field fluorescence microscopy," Opt. Lett. 24(14), 954-956 (1999).

2. H. Köhler, "On Abbe's theory of image formation in the microscope," J. Mod. Opt. 28(12), 1691-1701 (1981).

3. F. Bergermann et al., "2000-fold parallelized dualcolor STED fluorescence nanoscopy," Opt. Exp. 23 (1), 211-223 (2015).

4. T. Wilson, Confocal Microscopy, Vol. 426, pp. 1-64, Academic Press, London (1990).
5. T. Wilson, "Resolution and optical sectioning in the confocal microscope," J. Microsc. 244(2), 113-121 (2011).

6. Z. Rong et al., "Real-time super-resolution imaging by high-speed fluorescence emission difference microscopy," J. Mod. Opt. 61(16), 1364-1371 (2014).

7. C. Kuang et al., "Breaking the diffraction barrier using fluorescence emission difference microscopy," Sci. Rep. 3, 1441-1447 (2013).

8. Y. Fang et al., "Enhancing the resolution and contrast in CW-STED microscopy," Opt. Commun. 322, 169-174 (2014).

9. M. J. Rust, M. Bates, X. Zhuang, "Stochastic optical reconstruction microscopy (STORM) provides sub-diffraction-limit image resolution," Nat. Methods 3(10), 793 (2006).

10. E. Betzig et al., "Imaging intracellular fluorescent proteins at nanometer resolution," Science $\mathbf{3 1 3}$ (5793), 1642-1645 (2006).

11. S. W. Hell, J. Wichmann, "Breaking the diffraction resolution limit by stimulated emission: Stimulatedemission-depletion fluorescence microscopy," Opt. Lett. 19(11), 780-782 (1994).

12. R. Heintzmann, C. G. Cremer, Laterally modulated excitation microscopy: Improvement of resolution by using a diffraction grating, BiOS Europe'98, International Society for Optics and Photonics (1999).

13. M. G. Gustafsson, "Surpassing the lateral resolution limit by a factor of two using structured illumination microscopy," J. Microsc. 198(2), 82-87 (2000).

14. W. A. Carrington et al., "Superresolution three-dimensional images of fluorescence in cells with minimal light exposure," Science 268(5216), 1483-1487 (1995).

15. H. Kano et al., "Avalanche photodiode detection with object scanning and image restoration provides 2-4 fold resolution increase in two-photon fluorescence microscopy," Bioimaging 4(3), 187-197 (1996).

16. A. Sentenac et al., "High-resolution total-internalreflection fluorescence microscopy using periodically nanostructured glass slides," J. Opt. Soc. Am. A, Opt. Image Sci. 26(12), 2550-2557 (2009).

17. H. Dehez, M. Piché, Y. De Koninck, "Resolution and contrast enhancement in laser scanning microscopy using dark beam imaging," Opt. Exp. 21 (13), 15912-15925 (2013).

18. E. Rittweger, D. Wildanger, S. Hell, "Far-field fluorescence nanoscopy of diamond color centers by ground state depletion," Europhys. Lett. 86(1), 14001 (2009).

19. B. Harke et al., "Three-dimensional nanoscopy of colloidal crystals," Nano Lett. 8(5), 1309-1313 (2008). 
20. S. Li et al., "Enhancing the performance of fluorescence emission difference microscopy using beam modulation," J. Opt. 15(12), 125708 (2013).

21. A. Gasecka et al., "Resolution and contrast enhancement in coherent anti-Stokes Raman-scattering microscopy," Opt. Lett. 38(21), 4510-4513 (2013).

22. Z. Rong et al., "Super-resolution microscopy based on fluorescence emission difference of cylindrical vector beams," Opt. Commun. 354, 71-78 (2015).

23. S. Segawa, Y. Kozawa, S. Sato, "Resolution enhancement of confocal microscopy by subtraction method with vector beams," Opt. Lett. 39(11), 3118-3121 (2014).

24. X. Hao et al., "Effects of polarization on the deexcitation dark focal spot in STED microscopy," J. Opt. 12(11), 115707 (2010).
25. D. Wildanger et al., "A compact STED microscope providing 3D nanoscale resolution," J. Microsc. 236(1), 35-43 (2009).

26. S. You et al., "Three-dimensional super-resolution imaging for fluorescence emission difference microscopy," AIP Adv. 5(8), 084901 (2015).

27. S. Segawa, Y. Kozawa, S. Sato, "Demonstration of subtraction imaging in confocal microscopy with vector beams," Opt. Lett. 39(15), 4529-4532 (2014).

28. N. Wang, T. Kobayashi, "Polarization modulation for fluorescence emission difference microscopy," Opt. Exp. 23(10), 13704-13712 (2015).

29. N. Wang, T. Kobayashi, "Numerical study of the subtraction threshold for fluorescence difference microscopy," Opt. Exp. 22(23), 28819-28830 (2014). 